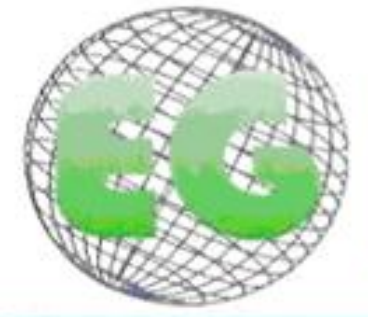

$N^{\circ} 40$

\title{
El cuidado de enfermeria ante los procesos quirúrgicos estéticos
}

Nursing care to the aesthetic surgical procedures

\section{*Laguado Jaimes, Elveny ${ }^{* *}$ Yaruro Bacca, Karol **Hernández Calderón, Emily Jurani}

\author{
*Magister en Enfermería.Docente Facultad de Enfermería. Universidad Cooperativa de Colombia. \\ E-mail: Elveny.Laguado@campusucc.edu.co ** Estudiante Último Semestre. Facultad de Enfermería. \\ Universidad Cooperativa de Colombia. Colombia.
}

Palabras clave: cuidados posoperatorios; cirugías estéticas; enfermería: ansiedad; dolor.

Keywords: postoperative care; plastic surgery; nursing; anxiety; pain.

\section{RESUMEN}

Introducción: El cuidado de enfermería dado en diferentes situaciones no puede estar ajeno al auge de las cirugías estéticas y su relación con la construcción social y cultural del significado del cuerpo, y las respuestas humanas de las personas que deben ser intervenidas. Desde algunas perspectivas sociológicas se describe la influencia de los paradigmas estéticos sobre los ideales y las prácticas de transformación actuales que se realizan los individuos para alcanzar la belleza que se instaura en la sociedad actual.

Objetivo: Fundamentar las intervenciones de enfermería para el manejo de los síntomas desagradables en el posoperatorio de cirugías estéticas, de igual forma determinar las causas para realizarse los procedimientos.

Método: Revisión bibliográfica y documental en bases de datos SciELO, Scopus, SCience Direct; se utilizan palabras claves como: cirugía, estética, educativo, ansiedad, dolor, cirugía plástica, cuidados, proceso quirúrgico, posoperatorio, preoperatorio, educación, guía de atención, síntomas desagradables, enfermería.

Conclusiones: La cirugía estética es un campo emergente y poco abordado por parte de los profesionales de enfermería; entre las causas los estereotipos, el uso y la percepción que se tiene del cuerpo nunca se ha mantenido al margen de la propia condición corporal. La educación preoperatoria herramienta de enfermería para ayudar al afrontamiento del proceso en el posoperatorio; así como el apoyo social y familiar en esta persona es determinante, aumenta el afrontamiento y disminuye niveles de ansiedad y de igual forma brindar un proceso educativo a la familia. 


\section{ABSTRACT}

Introduction: Nursing care given in different situations, you cannot be oblivious to the rise of cosmetic surgery and its relation to the social and cultural construction of the meaning of the body, and the human responses of individuals to be tapped. Since some sociological perspectives influence the aesthetic paradigms on the ideals and practices of current transformation that individuals are made to achieve the beauty that is established in today's society is described.

Objective: To base nursing interventions to manage unpleasant symptoms in the postoperative cosmetic surgeries, similarly determine the reasons for the procedures performed.

Methods: Literature review and documentary databases Scielo, Scopus, Science Direct data; surgery, aesthetic, educational, anxiety, pain, plastic surgery, care, surgical procedure, postoperative, preoperative, education, care guide, unpleasant symptoms, nursing: key words are used.

Conclusions: Cosmetic surgery is an emerging field and little touched by nurses; between cause's stereotypes, the use and perception of the body has never been kept out of the BCS itself. Education preoperative nursing tool to aid the coping process in the postoperative period; as well as social and family support is crucial in this person, increases coping and decreases anxiety levels and likewise provide an educational process for the family.

\section{INTRODUCCIÓN}

En el mundo el auge de la cirugía plástica es un hecho sin precedentes, se realizan cada año 234 millones de operaciones de cirugía mayor, lo que equivale a una operación por cada 25 personas. ${ }^{1}$ A su vez es un campo emergente, desconocido, poco explorado para la disciplina de enfermería. Enfermería requiere conocimientos teóricos y prácticos específicos para el cuidado de enfermería en el ámbito de la cirugía plástica, para brindar un cuidado integral en personas y dar respuesta a las necesidades de estos usuarios.

A nivel suramericano Argentina, Brasil y Colombia se han consolidado como plataformas y destinos para cirugías estéticas; estos procedimientos requieren una preparación diferente del usuario, desde la misma elección de la parte del cuerpo que se quiere cambiar y los efectos a nivel físico y emocional que pueden ocasionar los resultados del proceso realizado. Esta intervención quirúrgica genera reacciones emocionales agudas, los individuos que solicitan este tipo de intervenciones, experimentan un dualismo entre los rasgos físicos observables y algunos autoesquemas internalizados como la autoimagen, auto concepto y autoestima. ${ }^{2}$

Sin embargo es importante la comprensión del fenómeno social y cultural que conlleva a las personas a someterse a este tipo de procedimientos; el significado y percepción que adquiere el individuo de su corporalidad, la cual está sujeta a las formas, tamaños y simetrías perfectas que impone la cultura; el modelo estético predominante delgadez, conlleva a que las personas se sometan a todo tipo de procedimientos estéticos para lograr estar dentro de los parámetros de la belleza cultural. $^{3}$

Ante los procesos quirúrgicos surgen necesidades físicas y emocionales evidenciadas como síntomas desagradables, los cuales su intensidad, depende de la complejidad del acto quirúrgico, duración del mismo, pericia del cirujano, existencia o no de iatrogenias, idiosincrasia del paciente, capacidad de afrontamiento de cada persona; lo anterior influye en la recuperación. El estudio de España concluye que los efectos 
beneficiosos se logran en la medida en que la educación se adapte según la ansiedad. ${ }^{4}$

En enfermería el cuidado en cirugías estéticas debe orientarse al manejo de los síntomas desagradables en la fase posoperatoria, como son el dolor, la inflamación, la angustia, el stress y el miedo, experimentados por los usuarios como los más comunes. ${ }^{5}$ Las intervenciones orientadas a que el usuario se adapte a este nuevo cambio, así se cumplirá un objetivo fundamental en el cuidado de enfermería basado en la atención con calidad y tres principios fundamentales que cumplen un papel importante en el rol que desempeña día a día la enfermera, eficaz, eficiente y oportuno. ${ }^{6}$

Mediante la revisión se busca indagar sobre los cuidados pre y posoperatorios realizados en cirugías estéticas, que se constituyan en la base para orientar y protocolizar el cuidado de enfermería, evitando la variabilidad de la atención y orientada en la disminución de los síntomas desagradables. ${ }^{7}$

\section{MÉTODOS}

Mediante esta investigación se realizó una revisión sistemática de estudios publicados en bases de datos entre los años 2009 y 2014 teniendo en cuenta, la selección del tema relacionado con las generalidades de la cirugía estéticas, la ansiedad en procedimientos quirúrgicos, cuidados emocionales y cuidados en el pre y posoperatorios y tipo cirugías.

Las fuentes de la búsqueda se tomó de la base de datos: SciELO, Scopus, Lilacs, SCienceDirect entre otras, se utilizaron palabras claves como: cirugía, estética, educativo, ansiedad, dolor, cirugía plástica, cuidados, proceso quirúrgico, posoperatorio, preoperatorio, educación, guía de atención, síntomas desagradables, enfermería, trastorno dismórfico.

Los criterios de selección de los artículos encontrados estuvieron determinados por los objetivos de la investigación; inicialmente se revisa el título, el resumen y los resultados expuestos en cada uno de los estudios; respecto al título se puede observar si es útil y relevante para el tema de investigación, el resumen permitió analizar el contenido del mismo y si los resultados que son de gran importancia nos pueden mostrar si estos son aplicables al tema de estudio de esta investigación.

Después se realizó la lectura crítica de los documentos para excluir los artículos científicos que no exponen de manera clara el tema de estudio de la investigación y por otra parte incluir en esta revisión aquellos estudios que aportan científicamente en el rol profesional de enfermería en el cuidado de estos pacientes.

Los estudios que hacen parte de esta revisión cumplieron con los siguientes criterios:

- Describir el manejo de los síntomas desagradables: dolor y ansiedad en proceso quirúrgicos estéticos y reconstructivos

- Manejo interdisciplinario de pacientes de cirugías estéticas y reconstructivas en fases del pre y posoperatoria de cirugías estética y reconstructiva. 
- Describir los cuidados de enfermería enfocados al manejo de los síntomas desagradables en proceso quirúrgicos estéticos y reconstructivos

También es preciso determinar los criterios de exclusión que fueron tomados en cuenta para esta investigación los cuales corresponden a: Estudios de investigación que no referencian claridad en cuanto a las intervenciones realizadas en el manejo de síntomas desagradables en proceso quirúrgicos estéticos y estudios en los que se determina no hubo cumplimiento de los criterios éticos mínimos para el desarrollo de la investigación.

Entre las limitantes de la búsqueda se encontró que enfermería no ha indagado mucho en la cirugía estética y por ende son muy pocos los artículos disponibles en los cuales enfermería intervenga directamente en el usuario de cirugías estéticas.

\section{RESULTADOS}

Se revisaron 83 documentos, de los de los cuales 72 fueron artículos de investigación 4 tesis de grados y 7 publicaciones de sitios web y periódicos; estos últimos fueron descartados, puesto que la revisión se limita a los artículos de investigación. De los 72 artículos que hablan de cirugía plástica y similar se encontraron 12 mencionaban las generalidades de la cirugía estética, auge y causas. 6 hablaban de cirugías abdominales y de estómago; 7 artículos de ansiedad preoperatoria y sus efectos, 2 de comunicación preoperatoria, 5 de dolor, 4 de cirugía glúteos, 6 de mamas, 6 posoperatorio, 15 artículos de cuidados de enfermería en las diferentes fases de las cirugías y 9 de cirugía reconstructiva, los cuales se descartan por estar delimitado a estéticos. De las 63 revisiones bibliográficas se encontraron 15 de Colombia, 12 de España, 14 de USA, 7 de México, 5 de Brasil, 3 de Venezuela, 2 de Chile, Cuba, y Corea y 1 de Ecuador, Argentina, Salvador, Bolivia y Australia.

Los idiomas en los que se realizó la búsqueda fue en español donde se obtuvo 45 artículos, en inglés con 13 y en portugués con 5 publicaciones; corresponden al período de 2009 a 2014.

\section{Desarrollo}

\section{Causas de la Cirugía Estética:}

En la revisión se encuentra como aspecto fundamental, que la belleza en las personas está delimitada por el estereotipo que imponga la cultura sobre las características fenotípicas de una persona atractiva para la sociedad. Como atractivos los hombres y mujeres deben ser jóvenes, blancos, con los ojos, el cabello y la piel de tonos claros, la nariz fina y respingada y la forma ovalada de la cara. En cuanto al peso se ha vendido una imagen de delgadez en las mujeres y en los hombres la musculatura y su definición física; las necesidades de aceptación de las personas en las sociedades, sin importar todos los efectos secundarios y el costo-beneficio que puede generar un procedimiento estético, se constituyen en la búsqueda de la belleza ${ }^{8}$

De igual forma en México, la belleza y estética está en el prototipo de la mujer bella, una joven con edad promedio de 25 años, entre 21 y 28 años, con peso inferior al normal. La obesidad la perciben como un estigma social, por lo cual las personas se 
someten a prácticas quirúrgicas y obtener la figura bella aceptada por una sociedad. En México el procedimiento estético de más auge es la blefaroplastia (cirugía de párpados) y la implantación de senos. $14.41 \%$ se practica a mujeres menores de 21 años y $58.70 \%$ a personas con edad entre los 21 y 50 años. ${ }^{5}$

Así mismo en las ciudades de Colombia, Bogotá, Barranquilla y Medellín las personas que más cirugías estéticas se realizan, están motivadas por aparentar una imagen delgada y así sentirse más cómodas con su cuerpo y consigo mismas, confirmando que la imagen corporal es una de las razones principales para someterse a un procedimiento estético. Igualmente reporto que entre las preocupaciones mayores eran los efectos secundarios y el manejo del posoperatorio de estos eventos ${ }^{9}$.

De igual manera el culto al cuerpo conlleva a tener como opción la cirugía estética, debido a que permiten transformar de forma relativamente rápida la parte del cuerpo que se desea mejorar. Según un estudio realizado en Colombia, muestra que las mujeres sueñan con un cuerpo armonioso y simétrico; por ende se someten a una cirugía estética para corregir la asimetría que para ellas puede verse en la nariz, su busto, el abdomen e incluso en sus glúteos. La familia, la pareja, el trabajo y los medios de comunicación han influido de manera importante en ese ideal de belleza; sometiéndose a la cirugía estética para lograr la aceptación de la sociedad de la cual hacen parte ${ }^{10}$.

Entre las principales motivaciones de los pacientes para intervenir su cuerpo están relacionadas con la autoestima, felicidad, la satisfacción consigo mismo, el éxito, mejoría en las relaciones sociales y laborales. En el estudio de Venezuela realizan una revisión sobre el éxito de las personas bellas físicamente, concluyeron que la gente atractiva físicamente es más exitosa, beneficiando su vida social como la laboral; la percepción de imperfecciones o defectos en la apariencia física se considera como factor esencial en las interrelaciones humanas, asociada al éxito profesional y social siendo la imagen corporal una puerta que abre o cierra oportunidades $^{11}$.

Aunque se ha observado una mejoría de la calidad de vida, la autoestima y el estado de ánimo, el auge de enfermedades psiquiátricas como el trastorno dismórfico corporal y el trastorno depresivo con mayor incidencia en la adolescencia tardia, en las personas que se van a someter a una cirugia plastica, se constituye en una situación que requiere la competencia de psiquiatria ${ }^{12}$.

\section{El dolor en Cirugía Estética:}

Pero como todo procedimiento quirúrgico, los efectos que se presentan requieren de cuidados, por consiguiente enfermería en compañía del equipo de trabajo deben procurar realizar una atención adecuada que permita controlar el dolor y demás síntomas desagradables para que la experiencia de la cirugía estética se convierta en una experiencia manejable y satisfaga las expectativas de la persona que se somete a ella.

En las revisión, la prevalencia del dolor en el posoperatorio de las cirugías es el más relevante de los efectos, puesto que desencadena eventos que complican la rehabilitación de los usuarios; para lo anterior es importante definir el dolor como una experiencia perceptual y afectiva compleja, determinada tanto por las respuestas biológicas a los estímulos nociceptivos como por el significado subjetivo de esos 
estímulos para cada persona. Al ser persistente e intenso con efectos potencialmente dañinos que se manifiestan en el ámbito de diversos sistemas y con una respuesta neuroendocrina generalizada ${ }^{13}$

Evidencia de lo anterior, el estudio realizado en un hospital de México encontró que la prevalencia del dolor era mayor al $60 \%$ a pesar de la analgesia previa realizada; de los cuales el $40 \%$ presentaban dolor severo y fuerte, llegando a la conclusión de que el papel de la anestesia no se cumple con efectividad y la analgesia posoperatoria no cumple con los fines generales, como consecuencia se encuentran la prevalencia del dolor, donde el primer día de posoperatorio es de un riesgo elevado a padecer eventos no deseados ligados a la presencia de síntomas desagradables y el no control de los mismo ${ }^{14}$.

De igual forma, la importancia del dolor agudo postoperatorio radica en su alta frecuencia, en su inadecuado tratamiento y en las repercusiones que tiene en la evolución y en la recuperación del paciente. En España, independientemente del tipo de intervención, el $30 \%$ de los pacientes experimentan dolor leve tras la cirugía, el $30 \%$ dolor moderado y el $40 \%$ dolor severo. La analgesia que se proporciona debe estar acorde a sus características individuales y el personal de enfermería debe estar en la capacidad de realizar una valoración subjetiva del dolor y realizar la intervención adecuada para proporcionar un cuidado integral al usuario que se somete a cualquier cirugía y en especial a la estética ${ }^{15}$.

Del mismo modo, en el estudio realizado en Pereira encontraron que a pesar de la evidencia científica sobre el manejo del dolor, la mayoría de los pacientes en las 4 primeras horas no estaba controlado y una proporción menor tampoco a las 24 horas del postoperatorio, otros pacientes durante todo el intervalo de tiempo no lograron niveles aceptables de control de dolor. Además, un porcentaje significativo de pacientes exigió analgesia de rescate a consecuencia de la intensidad del dolor que estaban percibiendo ${ }^{16}$.

Las consecuencias de experimentar dolor de manera intensa y de manera prolongada, altera la calidad de vida como la cicatrización de las heridas. Un control adecuado del mismo contribuye también a que las heridas cicatricen más rápidamente. Los pacientes necesitan eliminar el dolor o al menos reducirlo de forma significativa, sin producir efectos psicológicos secundarios adversos tales como la reducción de los niveles de actividad ${ }^{17}$.

\section{Ansiedad en Cirugía Estética:}

Otra de las manifestaciones en las cirugías, es la ansiedad, condición emocional transitoria consistente en sentimientos de tensión, aprehensión, nerviosismo, temor y elevada actividad del sistema nervioso autonómico. La cirugía es percibida como un agente estresor importante, desde una perspectiva biopsicosocial y educativa, se puede controlar.

La ansiedad conlleva casi siempre a un estado emocional negativo y activación fisiológica provocando que el organismo se prepare para afrontar el peligro percibido; en la revisión un estudio en España, orientado a determinar qué estrategias son las adecuadas en el ámbito hospitalario para disminuir los niveles de ansiedad en los pacientes pre quirúrgicos, establece que se requiere un trato personalizado, un modelo de comunicación adecuado entre el personal sanitario y los pacientes, 
enseñar al enfermo el manejo del locus de control interno y entender la infraestructura y la rutina hospitalaria a partir de las necesidades de los usuarios ${ }^{18}$.

El paciente que va a ser intervenido quirúrgicamente se encuentra en una realidad que para él puede ser percibida como una amenaza, y que por tanto, esto puede originar un sentimiento de ansiedad inevitable, por esto el proporcionar apoyo emocional e información completa del procedimiento y sus complicaciones, es fundamental para permitir potenciar la expresión de los sentimientos de angustia previos a la intervención quirúrgica, en un estudio se analizan los efectos de intervenciones específicas, como la visita preoperatoria para disminuir la ansiedad del paciente antes de la cirugía ${ }^{19}$.

Como intervención de enfermería, mediante un ensayo clínico con dos grupos, al dar información al paciente y familia del proceso quirúrgico, determina si puede beneficiarles o por el contrario les genera más ansiedad; con un grupo experimental se aplicó la intervención enfermera en el postoperatorio temprano y con otro la intervención enfermera antes de ser operado y los resultados fueron satisfactorios, la información brindada antes, benefició al usuario y su familia y disminuyó la carga de ansiedad en el mismo ${ }^{20}$.

\section{Cuidados de Enfermería:}

El incremento de procedimientos estéticos en el mundo conlleva a que sea un campo más en el cual enfermería debe poseer las herramientas prácticas y conceptuales que garanticen la calidad del cuidado que brinda. ${ }^{3}$ Por otro lado las intervenciones preoperatorias dan el inicio a un plan de cuidados que debe recibir una persona que se somete a una cirugía estética, es ahí donde mediante el proceso educativo por enfermería, contribuye con la disminución de los niveles de ansiedad ${ }^{20}$.

Otros estudios relacionados, caracterizan la atención de enfermería en la fase preoperatorio reconociendo las necesidades del paciente y la atención adecuada, orientada sobre las posibles experiencias de los síntomas en varios contextos y brindar información útil diseñando métodos efectivos que reduzcan los síntomas desagradables. 21

El proceso de enfermería es el método mediante el cual se aplica una base teórica y científica al ejercicio profesional, la trascendencia de la profesión de enfermería radica en la cientificidad de sus intervenciones basadas en criterios técnicos, objetivos y medibles. Estandarizando cuidados a través de las taxonomías NANDA, NOC, NIC contribuye al cuidado de enfermería de pacientes quirúrgicos.

En el estudio realizado en Cuenca, Ecuador, encontraron que con la aplicación del P.A.E. en el área de cirugía, el $70 \%$ del personal profesional aplica parcialmente las cinco etapas del proceso y el 30\% aplica solo tres de las cinco etapas de proceso; concluyen que los usuarios del área de cirugía se ven afectados debido a la omisión del proceso de atención de enfermería, como consecuencias complicaciones y mayor tiempo de estancia hospitalaria ${ }^{22}$.

De acuerdo con la revisión de estudios relacionados con los cuidados de enfermería, los cuáles se les asigna una denominación de acuerdo a la taxonomía NIC, en el cuadro siguiente: 
Tabla 1. Hallazgos de Cuidados de Enfermería según estudios y Etiqueta NIC.

\begin{tabular}{|c|c|}
\hline Cuidados de Enfermería según estudios & Etiqueta NIC * \\
\hline $\begin{array}{l}\text { Evaluar al paciente y obtener una historia clínica } \\
\text { completa que permita identificar cualquier tipo de } \\
\text { alteración potencial de complicación ante la intervención } \\
\text { quirúrgica estética. }{ }^{23}\end{array}$ & $\begin{array}{l}\text { 2880:Coordinación } \\
\text { preoperatoria } \\
7920: \text { Documentación }\end{array}$ \\
\hline $\begin{array}{l}\text { Mediante las intervenciones de enfermería } \\
\text { fundamentadas científicamente en el proceso de atención } \\
\text { de enfermería, orientadas a las necesidades del paciente } \\
\text { y sus familiares disminuye así los factores de riesgo } \\
\text { afectivos y cognitivos, que pueden impactar las } \\
\text { experiencias de los síntomas, por ello debe personalizar } \\
\text { el cuidado de enfermería de acuerdo a las características } \\
\text { individuales del paciente }{ }^{24} \text {. }\end{array}$ & $\begin{array}{l}\text { 5270: Apoyo emocional } \\
\text { 5240: Asesoramiento } \\
\text { 5510: Educación sanitaria }\end{array}$ \\
\hline $\begin{array}{l}\text { Una visita preoperatoria de enfermería programada con } \\
\text { tiempo, los resultados son positivos sobre el nivel de } \\
\text { ansiedad, el autocontrol de miedo y ansiedad, los } \\
\text { conocimientos de cuidados de enfermedad, el nivel de } \\
\text { comodidad y en nivel de dolor; generando en el paciente } \\
\text { un alto grado de satisfacción }{ }^{25}\end{array}$ & $\begin{array}{l}\text { 5510: Educación sanitaria } \\
\text { 5240: Asesoramiento } \\
\text { 5606:Enseñanza } \\
\text { individual } \\
\text { 5820: Disminución de la } \\
\text { ansiedad } \\
\text { 1400:Manejo del dolor } \\
\text { 2880:Coordinación } \\
\text { preoperatoria }\end{array}$ \\
\hline $\begin{array}{l}\text { La intervención de enfermería pre quirúrgica realizada } \\
\text { una hora antes de la intervención quirúrgica no logra } \\
\text { disminuir los niveles de ansiedad del usuario; no facilita el } \\
\text { efecto ansiolítico }{ }^{26} \text {. }\end{array}$ & $\begin{array}{l}\text { 2880:Coordinación } \\
\text { preoperatoria }\end{array}$ \\
\hline $\begin{array}{l}\text { Disponer de un protocolo de cuidados peri operatorios de } \\
\text { enfermería en cirugía plástica asegura la estandarización } \\
\text { de la práctica de enfermería y aumenta la calidad de los } \\
\text { cuidados y la seguridad de las pacientes en su domicilio. }\end{array}$ & $\begin{array}{l}\text { 2880:Coordinación } \\
\text { preoperatoria }\end{array}$ \\
\hline $\begin{array}{l}\text { El procedimiento quirúrgico ocasiona al paciente y su } \\
\text { familia una serie de alteraciones en su comportamiento } \\
\text { que pueden ser aliviadas con la preparación emocional al } \\
\text { paciente y a su familia, con una explicación clara sobre el } \\
\text { procedimiento que se le va a practicar y las condiciones } \\
\text { en que se encontrará después de la cirugía; } \\
\text { disminuyendo los niveles de ansiedad. }\end{array}$ & $\begin{array}{l}\text { 5270: Apoyo emocional } \\
\text { 5240: Asesoramiento } \\
\text { 5820: Disminución de la } \\
\text { ansiedad }\end{array}$ \\
\hline
\end{tabular}


La comunicación terapéutica, permite brindar información a los pacientes para que aprendan, entiendan y busquen una solución a su malestar a través de la construcción y la reestructuración de la información, favoreciendo la comprensión del paciente en las diversas situaciones, minimizando $o$ evitando los errores $y$ efectos no deseados. $^{7}$

El cuidado emocional implica una actitud empática que predispone a la enfermera a captar los sentimientos, deseos, intereses y necesidades de la persona que cuida, para vivir en cierto modo su experiencia, sin confundir nuestros propios sentimientos" 23 .

La preparación preoperatoria basada en la consideración particular de las estrategias de afrontamiento. Se ha evidenciado en estudios que las personas que recibieron programas de afrontamiento presentaron mayor disminución en la ansiedad, menor nivel de dolor percibido y una baja frecuencia de complicaciones.

Durante el posoperatorio inmediato que transcurre desde la recuperación anestésica hasta las 24 horas, los cuidados de enfermería se dirigen a restablecer el equilibrio fisiológico, aliviar el dolor y prevenir complicaciones. Se pueden aplicar medidas no farmacológicas como la relajación, masaje, aplicación de frio o calor para complementar los medicamentos. ${ }^{27}$

El posoperatorio mediato que va desde las 24 hasta las 72 horas, la valoración cuidadosa ayuda al paciente a su recuperar su funcionamiento normal con mayor rapidez. Realizar vigilancia de signos vitales, estado respiratorio ante los efectos de los anestésicos, nivel del dolor, integridad de la herida, consumo oral, estado nutricional, ruidos intestinales, distensión abdominal, flatos, heces, equilibrio de líquidos, hematocrito, hemoglobina, como factores críticos que influyen en la recuperación del paciente. Se debe observar sangrado, tipo e integridad del vendaje y drenes, la tolerancia a la actividad, capacidad para participar en la atención, pues son el resultado de las actividades de enseñanza específica. ${ }^{27}$
5606:Enseñanza

individual

5270: Apoyo emocional

5270: Apoyo emocional

5270: Apoyo emocional

5230:Aumentar el afrontamiento

1400:Manejo del dolor

6680:Monitorización de signos vitales

1400:Manejo del dolor 6680:Monitorización de signos vitales

3660:Cuidados de las heridas

3590:Vigilancia de la piel

Elaboración propia

Identificando las necesidades de apoyo a los usuarios y brindando educación ayuda a disminuir los síntomas desagradables en la fase posoperatoria; por consiguiente brindar información preoperatoria disminuye la ansiedad del paciente, facilitando su recuperación y disminuyendo la estancia hospitalaria ${ }^{27}$. 


\section{DISCUSIÓN}

En relación a la revisión realizada, los estudios mencionados ${ }^{5,8,9,10,11,12}$, identifican entre las principales causas para optar por la cirugía estética, los estereotipos definidos por la cultura que se consideran como belleza, los cuales aumentan la autoestima, estado de ánimo y calidad de vida de quienes eligen estos procedimientos, sin embargo, es importante considerar la dimensión psicológica, puesto que no es suficiente, cuando la persona adicional a la necesidad de verse bién físicamente, presenta trastornos psiquiátricos que modifican su perspectiva ante los resultados.

Como efecto el dolor según estudios revisados ${ }^{13,14,15,16,17}$, su manejo, las guías del control del dolor implementadas en algunas instituciones y la analgesia realizada en cada procedimiento, son insuficientes. Cabe resaltar que en el posoperatorio de cirugías estéticas se debe realizar un manejo interdisciplinario del dolor para favorecer la recuperación del usuario y que la experiencia emocional del procedimiento no sea contraproducente para la rehabilitación del paciente.

Ahora en relación a la ansiedad, en lo expuesto anteriormente, según estudios ${ }^{18,19,20}$, la disminución de la ansiedad genera en los pacientes mayor seguridad en sentirse más recuperados, por tal motivo el paciente quirúrgico sufre el menor dolor posible, consume menos analgésicos, se encuentra lo más tranquilo posible. Lo más importante es reducir la ansiedad mediante la educación que aclare dudas a los pacientes de ciertos puntos que para ellos son importantes.

Los cuidados de enfermería cumplen una función muy determinante; en los diversos estudios analizados $3,7,20,21,22,23,24,25,26,27$, se proponen diagnósticos de enfermería para el inicio de protocolos de planes de cuidado en cirugías estéticas; fundamental destacar la visita preoperatoria de enfermería programada con el fin de recolectar los datos del usuario por medio de la entrevista, diligenciamiento de la documentación necesaria, valoración cefalocaudal del usuario y la identificación de cualquier factor de riesgo que pueda ser una complicación mayor con el desarrollo de la cirugía estética.

En el cuidado de enfermería es importante tener en cuenta desde el patrón empírico el conocimiento de los factores fisiológicos que están relacionados con los sistemas orgánicos normales, las patologías y los niveles de nutrición, en las cirugías estéticas son realizadas de acuerdo a las características de la persona y no existe un patrón de medida de la cirugías, estas se dan de acuerdo a la tolerancia del cuerpo en el que se realiza y los síntomas desagradables pueden presentarse de manera más agresiva en algunas personas que en otras, es de allí que cabe mencionar el cuidado individualizado y que no se debe estandarizar el tratamiento. Los factores psicológicos que se asocian con el estado anímico, la personalidad y el grado de ansiedad, estos procedimientos también se asocian a un trastorno dismórfico o un trastorno de conducta.

\section{CONCLUSIONES}

La cirugía estética es un campo emergente y poco abordado por parte de los profesionales de enfermería, reconocer que entre las causas están los estereotipos, el uso y la percepción que se tiene del cuerpo, lo cual nunca se ha mantenido al margen de la propia condición corporal; el tener o no sobrepeso, el estar o no en el la 
edad juvenil y el ser hombre o mujer son rangos que condicionan la percepción que se tiene del cuerpo y a la vez la percepción que tienen los demás.

En personas que son ansiosas y depresivas crónicas, se debe brindar una atención de mayor cuidado puesto que si los resultados de la cirugía no son los esperados este evento puede desencadenar una crisis emocional. La educación preoperatoria en una herramienta de enfermería debe utilizar para ayudar a que este tipo de usuarios incrementen el afrontamiento previniendo la manifestación de estos síntomas y garantizando una atención con calidad.

Durante el proceso quirúrgico, indiferente del tipo de cirugía, los cuidados de enfermería tienen una importancia fundamental en la disminución de riesgos, lograr el bienestar, la recuperación del paciente, y en la aceptación de las secuelas que se puedan derivar del procedimiento quirúrgico. El proceso de atención de enfermería es un método para que la enfermera realice la valoración, diagnóstico de enfermería, planeación ejecución y evaluación, cuya aplicación da como resultado un plan de cuidados.

Es importante para enfermería evaluar la subjetividad del usuario, debido a que la manifestación de síntomas como el dolor y la ansiedad van ligados a su parte psicológica, fisiológica y la situación en que se encuentre el usuario y no siempre se relaciona con el tipo de procedimiento estético, por ende enfermería debe hacer una intervención individualizada que permita realizar un cuidado integral al paciente que se somete a este tipo de procedimientos.

\section{REFERENCIAS}

${ }^{1}$ Olmedo Luceron MC, Sanchidrian de Blas C, Pla Maestre R, Puentes Gonzales MD, Rodriguez Perez P. Sistemática para la protocolización del cuidado de enfermería. Revista de calidad asistencial 2010 ( Citado 11 Ene 2014); 268-274. Disponible en: http://zl.elsevier.es/es/revista/revista-calidad-asistencial-256/sistematica-

protocolizacion-los-cuidados-enfermeria-13155588-originales-2010

${ }^{2}$ Betancur Zequeira DM. Atención de enfermería estandarizada para pacientes atendidos en la Unidad Quirúrgica de la Clínica. Revista de Calidad Asistencial. (2010). ( Citado 11 Ene 2014);25(5): 268-274. Disponible en: bvs.sld.cu/revistas/infd/n1412/infd031412.htm

${ }^{3}$ Espitia Cruz L C. El cuidado de enfermería en el contexto de la cirugía plástica estética: una reflexión a partir de los patrones de conocimiento. Umbral Científico 2009 ( Citado 11 Enero 2014); (15): 8-14. Disponible en: http://www.redalyc.org/articulo.oa?id=30415144002.

${ }^{4}$ Acuña Bermudez E. A, Serrano Castañeda SM, Buelvas Barrios CI, Luna Venegas SI, Perez Martinez SF, Avila Pacheco AM," etal."Estructuración psíquica en mujeres con múltiples Cirugías estéticas. Revista de Psicoanalisis, Psicoterapia y Salud Mental 2010 (Citado 12 Ene 2014); 3(7): 1-35. Disponible en: http://psi.usal.es/rppsm/n72010/acunabermudez.pdf

${ }^{5}$ Eliott, A. Plastica extrema: auge de la cultura de la cirugia estetica. Anagramas Enero-junio de 2011 (Citado 12 Ene 2014); 9(18): 145-164. Disponible en: http://www.scielo.org.co/pdf/angr/v9n18/v9n18a12.pdf

${ }^{6}$ Figueroa Varela MD. La opción de la reconfiguración estética: estudio en jóvenes universitarias. Revista de Estudios de Género. La ventana 2013 (Citado 12 Ene 2014); 4(37): 324-360. Disponible en: http://www.redalyc.org/articulo.oa?id=88428978013 
${ }^{7}$ Magdalena Diaz ML, Caragol Urgelles L, Sole Magdalena A, Fueyo Lorente A, Sopena Zubiria LA, Camporro Fernandez D. Desarrollo e implementación de un protocolo perioperatorio de enfermeria. cirugia plastica ibero-latinoamericana 2014 ( Citado 13 Agost 2014); 40(1): 21-28. Obtenido de http://scielo.isciii.es/pdf/cpil/v40n1/original3.pdf

${ }^{8}$ Ochoa Avalos C. La añoranza de la eterna juventud. La ventana .Julio-Diciembre de 2009 (Citado 12 Agost 2014); 4(34): 255-259. Disponible en: http://www.scielo.org.mx/pdf/laven/v4n34/v4n34a12.pdf

${ }^{9}$ Cabarcas Acosta KL, Londoño Pérez C. Trastornos de la conducta alimentaria, imagen corporal, afrontamiento. Psicologia y salud 2014 (Citado 12 Agost 2014); 24(2): 199-219.

Disponible

en:

http://revistas.uv.mx/index.php/psicysalud/article/view/925/1705

${ }^{10}$ Ibáñez Pabon JI.Sueñan con un cuerpo armonioso: motivaciones de las mujeres para realizarse procedimientos estéticos no reconstructivos. Revista de Educacion Fisica 2014 ( Citado 12 Agost 2014); 3(1): 56-88. Disponible en: http://aprendeenlinea.udea.edu.co/revistas/index.php/viref/article/viewFile/18929/1616 ${ }^{11}$ Pérez Bravo, A. El cuerpo objeto y la belleza sujeto. Omnia(Versión impresa) 2012 (Citado 12 Agost 2014); 18(3): 66-80. Disponible en: http://www.redalyc.org/pdf/737/73725513008.pdf

${ }^{12}$ Carreño Alvarez M. Importancia en la detección de trastorno dismórfico y depresión antes de someterse a un procedimiento estético electivo. Med UNAB 2009 (Citado 12 Ene 2014); 12: 46-51. Disponible en: http://revistas. unab.edu.co/index.php?journal=medunab\&page=article\&op=view\&path $\% 5 \mathrm{~B} \% 5 \mathrm{D}=50$ \&path $\% 5 \mathrm{~B} \% 5 \mathrm{D}=47$

${ }^{13}$ Gallardo J. (2010). Dolor postoperatorio: asado, presente y futuro. Rev Chil Anest 2010 (Citado 12 Ene 2014); 39: 69-75. Disponible en: http://www.sachile.cl/upfiles/revistas/4c5c17345914e_gallardo2.pdf

${ }^{14}$ Sada Ovalle T, Delgado Hernandez E, Castellanos Olivares A. Prevalencia del dolor en cirugia electiva de pacientes del hospital de especialidades del Centro Médico Nacional Siglo XXI IMSS. Revista Española del Dolor 2011 ( Citado 12 Agost 2014); 18(2): 91-97. Disponible en: http://revista.sedolor.es/pdf/2011_02_04.pdf

${ }^{15}$ Muñoz J M. Manual de dolor agudo postoperatorio.Barcelona Madrid: Ergon ; 2010.

${ }^{16}$ Machado Alba JE, Quintero AM, Mena García MF, Castaño Carmona CA, Lopez Saldarriaga EM, Marín Zuluaga DC, "et al." Evaluacion del manejo del dolor posquirurgico en pacientes adultos en una clinica de tercer nivel en Pereira Colombia. INVESTIGACIONES ANDINAS 2012 ( Citado 12 Mayo 2014); 25(14): 547-559. Disponible en: http://www.scielo.org.co/pdf/inan/v14n25/v14n25a05.pdf

${ }^{17}$ Pinto B, Montoya T. Hipnosis para el control del dolor en pacientes con quemaduras. Ajayu 2010 (Citado 12 Mayo 2014); 8(2): 122-156. Disponible en: http://www.ucb.edu.bo/publicaciones/ajayu/v8n2/v8n2a6.pdf

${ }^{18}$ Gordillo Leon F, Arana Martinez JM, Mestas Hernandez L. Tratamiento de la ansiedad en paciente prequirúrgicos. Revista Clínica de Medicina de Familia ( revista en la Internet). 2011 Oct (Citado 12 Mayo 2014); 4(3): 228-233. Disponible en: http://scielo.isciii.es/scielo.php?script=sci_arttext\&pid=S1699-

695X2011000300008\&lng=es. http://dx.doi.org/10.4321/S1699-695X2011000300008

${ }^{19}$ Valenzuela Millan J, Barrera Serrano JR, Ornelas Aguirre JM. Ansiedad preoperatoria en procedimientos anestesicos. Cirugia y Cirujanos Marzo - Abril de 2010 (Citado 12 Mayo 2014) ; 78(2): 151-156. Disponible en: http://www.medigraphic.com/pdfs/circir/cc-2010/cc102h.pdf

${ }^{20}$ Pacheco del Cerro J, Portero Ferrer FJ.Ensayo clinio sobre la influencia de la informacion para la reduccion de la ansiedad en posoperatorio temprano a pacientes y familiares sometidos a una microcirugía. Reduca (Enfermería, Fisioterapia y 
Podología) Serie Trabajos Fin de Master 2010 ( Citado 12 Mayo 2014); 2 (1): 11681189. Disponible en: $\quad$ http://revistareduca.es/index.php/reducaenfermeria/article/viewFile/235/257

${ }^{21}$ Bouwman Christóforo B E, Siqueira Carvalho D. Cuidados de enfermagem realizados ao paciente cirúrgigo no período pré-operatório. Rev Esc Enferm USP(on line) 2009 ( Citado 12 Ene 2014); 43(1): 14-22.Disponible en: http://www.scielo.br/pdf/reeusp/v43n1/02.pdf

${ }^{22}$ Maldona Gutierrez ZA, Naula Galabay MN, Suquinagua Ludizaca BM. Aplicacion del proceso de enfermeria en la atencion a pacientes hospitalizados en cirugia del Hospital José Carrasco Arteaga.(thesis).Universidad de Cuenca Facultad de Ciencias Médicas Escuela de Enfermeria; $2010 . \quad$ Disponible en: http://dspace.ucuenca.edu.ec/handle/123456789/3591

${ }^{23}$ Irrutia $P$, Villarroel $M$. Cuidados emocionales que brindan los enfermeros a pacientes prequirurgicos.(thesis). UNIVERSIDAD NACIONAL DE CÓRDOBA FACULTAD DE CIENCIAS MÉDICAS ESCUELA DE ENFERMERÍA; 2009. Disponible en: http://www.enfermeria.fcm.unc.edu.ar/biblioteca/tesis/irrutia patricia.pdf ${ }^{24}$ Laguado Jaimes E, Gomez Diaz MP. Teoria del manejo de sintomas desagradables para el cuidado de enfermeria en cirugia. Enfermeria Global 2014 ( Citado 13 Agost 2014); 13(3): 326-337.Disponible

http://revistas.um.es/eglobal/article/view/eglobal.13.3.170201

${ }^{25}$ Orihuela Pérez I, Perez Espinosa JA, Aranda Salcedo T, Zafra Norte J, Jiménez Ruiz RM, Martínez García A, "et al". Visita preoperatoria de enfermería: evaluación de la efectividad de la intervención enfermera y percepción del paciente. Enfermería Clínica 2010 ( Citado 13 Agost 2014); 20(6): 349-354. Disponible en: http://dialnet.unirioja.es/servlet/ejemplar?codigo $=254953$

${ }^{26}$ Diez Álvarez E, Arantzazu A, Mar J, Alvarez U, Belaustegi A, Lizaur B, Larrañaga A, " etal." Efectividad de una intervención preoperatoria de enfermería sobre el control de la ansiedad de los pacientes quirúrgicos. Enfermería Clínica Enero 2012 (Citado 12 Agost 2014); 22(1): 18-26. Disponible en: http://zl.elsevier.es/es/revista/enfermeriaclinica-35/articulo/efectividad-una-intervencion-preoperatoria-enfermeria-90093446 ${ }^{27}$ Conceicao Santos M L, Stelio FD, Candido Alves P, Bonfim Melo I, Carvalho Fernandes A F. (julio-agosto de 2010). Comunicação terapêutica no cuidado préoperatório de mastectomia / Therapeutic communication in perioperative care of mastectomy. Rev Bras Enferm Agosto 2010 (Citado 12 Agost 2014); 63(4): 675-678. Disponible en: http://www.scielo.br/pdf/reben/v63n4/27.pdf

Recibido: 19 de noviembre 2014; Aceptado: 20 de diciembre 2014

ISSN 1695-6141

(C) COPYRIGHT Servicio de Publicaciones - Universidad de Murcia 